\title{
Estimates of Covering Type and the Number of Vertices of Minimal Triangulations
}

\author{
Dejan Govc ${ }^{1,2} \cdot$ Wacław Marzantowicz ${ }^{3}$ (D) Petar Pavešić ${ }^{1}$
}

Received: 6 October 2017 / Revised: 2 March 2019 / Accepted: 3 April 2019 / Published online: 17 April 2019

(c) The Author(s) 2019

\begin{abstract}
The covering type of a space $X$ is a numerical homotopy invariant which in some sense measures the homotopical size of $X$. It was first introduced by Karoubi and Weibel (in Enseign Math 62(3-4):457-474, 2016) as the minimal cardinality of a good cover of a space $Y$ taken among all spaces that are homotopy equivalent to $X$. We give several estimates of the covering type in terms of other homotopy invariants of $X$, most notably the ranks of the homology groups of $X$, the multiplicative structure of the cohomology ring of $X$ and the Lusternik-Schnirelmann category of $X$. In addition, we relate the covering type of a triangulable space to the number of vertices in its minimal triangulations. In this way we derive within a unified framework several estimates of vertex-minimal triangulations which are either new or extensions of results that have been previously obtained by ad hoc combinatorial arguments.
\end{abstract}

Keywords Covering type $\cdot$ Minimal triangulation $\cdot$ Lusternik-Schnirelmann category $\cdot$ Cup-length

Editor in Charge: János Pach

W. Marzantowicz: Supported by the Research Grant NCN Grant 2015/19/B/ST1/01458.

P. Pavešić: Supported by the Slovenian Research Agency Program P1-0292 and Grant J1-7025.

Dejan Govc

dejan.govc@gmail.com

Wacław Marzantowicz

marzan@amu.edu.pl

Petar Pavešić

petar.pavesic@fmf.uni-lj.si

1 Faculty of Mathematics and Physics, University of Ljubljana, Jadranska 21, 1000 Ljubljana, Slovenia

2 Institute of Mathematics, University of Aberdeen, Fraser Noble Building 162, Elphinstone Road, AB24 3EF Aberdeen, UK

3 Faculty of Mathematics and Computer Science, Adam Mickiewicz University of Poznań, ul. Umultowska 87, 61-614 Poznań, Poland 
Mathematics Subject Classification Primary 55M; Secondary 55M30 - 57Q15 . 57R05

\section{Introduction}

Many concepts in topology and homotopy theory are related to the size and the structure of the covers that a given space admits. Typical examples that spring in mind are the Lebesgue dimension and Lusternik-Schnirelmann category. Karoubi and Weibel [9] have recently introduced another interesting measure for the complexity of a space based on the size of its good covers.

Recall that an open cover $\mathcal{U}$ of $X$ is said to be a good cover if all elements of $\mathcal{U}$ and all their non-empty finite intersections are contractible. Karoubi and Weibel defined $\operatorname{sct}(X)$, the strict covering type of a given space $X$, as the minimal cardinality of a good cover for $X$. Note that $\operatorname{sct}(X)$ can be infinite (e.g., if $X$ is an infinite discrete space) or even undefined, if the space does not admit any good covers (e.g. the Hawaiian earring). In what follows we will always tacitly assume that the spaces under consideration admit finite good covers.

Strict covering type is a geometric notion and is not homotopy invariant, which led Karoubi and Weibel to define the covering type of $X$ as the minimal size of a good cover of spaces that are homotopy equivalent to $X$ :

$$
\operatorname{ct}(X):=\min \{\operatorname{sct}(Y) \mid Y \simeq X\}
$$

The covering type is a homotopy invariant of the space and is often strictly smaller than the strict covering type, even for simple spaces like wedges of circles [9, Exam. 1.3]. Karoubi and Weibel also proved a useful result [9, Thm. 2.5] that the covering type of a finite CW complex is equal to the minimal cardinality of a good closed cover of some CW complex that is homotopy equivalent to $X$. Furthermore, they computed exactly the covering type for finite graphs (i.e., finite wedges of circles) and for some closed surfaces (sphere, torus, projective space), while giving estimates for the covering type of other surfaces. Finally, they estimated the covering type of mapping cones, suspensions and covering spaces. With our methods we have been able to refine and improve their results, and even to correct the upper estimate of $\operatorname{ct}\left(\mathbb{R} P^{m}\right)$ in [9, Exam. 7.3] (cf. Corollary 3.6).

Good covers arise naturally in many situations, e.g., as geodesically convex neighbourhoods in Riemannian manifolds or as locally convex covers of polyhedra. Their main feature is that the pattern of intersections of sets of a good cover captures the homotopy type of a space. Specifically, let $N(\mathcal{U})$ denote the nerve of the open cover $\mathcal{U}$ of $X$, and let $|N(\mathcal{U})|$ be its geometric realisation. We may identify the vertices of $|N(\mathcal{U})|$ with the elements of $\mathcal{U}$ and the points of $|N(\mathcal{U})|$ with the convex combinations of elements of $\mathcal{U}$. If $\mathcal{U}$ is numerable, that is, if $\mathcal{U}$ admits a subordinated partition of unity $\left\{\varphi_{U}: X \rightarrow[0,1] \mid U \in \mathcal{U}\right\}$, then the formula

$$
\varphi(x):=\sum_{U \in \mathcal{U}} \varphi_{U}(x) \cdot U
$$


determines the so-called Alexandroff map $\varphi: X \rightarrow|N(\mathcal{U})|$, which has many remarkable properties. In particular we have the following classical result, whose discovery is variously attributed to Leray, Borsuk and Weil (see [8, Corr. 4G.3] for a modern proof).

Theorem 1.1 (Nerve Theorem) If $\mathcal{U}$ is a numerable good cover of $X$, then the Alexandroff map $\varphi: X \rightarrow|N(\mathcal{U})|$ is a homotopy equivalence.

As a consequence, a paracompact space admits a finite good cover if and only if it is homotopy equivalent to a finite (simplicial or CW) complex. In the literature one can find many variants of the Nerve theorem, which under different sets of assumptions show that the Alexandroff map is a homotopy equivalence, or a weak homotopy equivalence, or a homology equivalence, etc.

The idea of covering type provides an important link between good covers and minimal triangulations. In general, given a polyhedron $P$, one often looks for triangulations of $P$ with the minimal number of vertices. Again, there are many variants and aspects of the problem so we introduce the following systematic notation: given a compact polyhedron $P$, we denote by $\Delta(P)$ the minimal number of vertices in a triangulation of $P$, i.e.,

$$
\Delta(P):=\min \left\{\operatorname{card}\left(K^{(0)}\right)|| K \mid \approx P\right\} .
$$

If $P$ is a manifold then one is principally interested in combinatorial (or PL-) triangulations, i.e., triangulations in which the links of vertices are combinatorial spheres. Thus for every PL-manifold $M$ we define

$$
\Delta^{\mathrm{PL}}(M):=\min \left\{\operatorname{card}\left(K^{(0)}\right) \mid K \text { is a PL-triangulation of } M\right\} .
$$

Computing $\Delta$ or $\Delta^{\mathrm{PL}}$ is a hard and intensively studied problem of combinatorial topology — see Datta [6] and Lutz [10] for surveys of the vast body of work related to this question.

Every triangulation of a space $X$ gives rise to a good cover of $X$ by taking open stars of vertices in the triangulation. On the other hand, if $\mathcal{U}$ is a good cover of $X$, then $|N(\mathcal{U})|$ is homotopy equivalent to $X$ by the Nerve theorem. Therefore, whenever $X$ has the homotopy type of compact polyhedron we may introduce a homotopy analogue of $\Delta(P)$ as

$$
\Delta^{\simeq}(X):=\min \{\Delta(P) \mid P \simeq X\}
$$

Clearly, $\Delta^{\simeq}(K)$ is a lower bound for other invariants, since

$$
\Delta^{\simeq}(P) \leq \Delta(P),
$$

and if $M$ is a PL-manifold

$$
\Delta^{\simeq}(M) \leq \Delta^{\mathrm{PL}}(M) .
$$


On the other hand, $\Delta^{\simeq}(X)$ is directly related to the covering type.

Theorem 1.2 If $X$ has the homotopy type of a finite polyhedron, then

$$
\operatorname{ct}(X)=\Delta^{\simeq}(X) \text {. }
$$

Proof Let $\mathcal{U}$ be a good cover of $X$ of cardinality $\operatorname{ct}(X)$. The nerve $N(\mathcal{U})$ has $\operatorname{ct}(X)$ vertices, and $|N(\mathcal{U})| \simeq X$ by the Nerve theorem, which implies $\Delta^{\simeq}(X) \leq \operatorname{ct}(X)$. Conversely, if $K$ is a simplicial complex such that $|K| \simeq X$, then the cover of $|K|$ by open stars of vertices is a good cover of $|K|$, therefore $\Delta^{\simeq}(X) \geq \operatorname{ct}(X)$.

As a consequence, it is of great interest to find good lower estimates for $\operatorname{ct}(X)$ as they in turn give lower bounds for the size of minimal triangulations. On the other hand, it is easy to find examples where $\operatorname{ct}(P)$ is strictly smaller than $\Delta(P)$ (cf. [9, Exam. 1.3]). The upper estimates for $\operatorname{ct}(X)$ are therefore less relevant as a tool for the study of minimal triangulations. Indeed, upper estimates of $\operatorname{ct}(X)$ are usually obtained by finding explicit triangulations of $X$, while the lower estimates are based on certain obstructions. The latter is a natural setting for the methods of homotopy theory, and is one of the reasons why the relation with $\operatorname{ct}(X)$ is so useful.

It appears to be much harder to distinguish between $\operatorname{ct}(M)$ and $\Delta^{\mathrm{PL}}(M)$ when $M$ is a closed manifold. Borghini and Minian [3] have recently proved that with one exception $\operatorname{ct}(M)=\Delta^{\mathrm{PL}}(M)$ for all closed surfaces (both orientable and non-orientable). The only exception is the genus 2 orientable surface $T \# T$, as they prove that $\operatorname{ct}(T \# T)=9$ while $\Delta^{\mathrm{PL}}(T \# T)=10$, which means that the minimal triangulation of $T \# T$ has 10 vertices but there exists a 2 -dimensional complex on 9 vertices whose geometric realisation is homotopy equivalent to $T$ \# $T$. Since the covering type is a homotopy invariant, it would be of interest to find conditions under which the covering type of a closed manifold $M$ coincides with the minimal number of vertices in a triangulation of $M$.

The paper is organised as follows. In the next two sections we relate the LusternikSchnirelmann category and the cohomology ring of a space to the covering type and derive a series of lower estimates for the covering type. In the last section we study the effect that suspensions and wedge-sums have on the covering type, and give some useful upper and lower estimates for the covering type of a Moore space.

\section{LS-Category Estimates}

Recall the definition of the Lusternik-Schnirelmann (LS-)category of a space $X$. A subset $A \subseteq X$ is categorical if the inclusion $A \hookrightarrow X$ is homotopic to the constant map. Then $L S$-category of $X$, denoted cat $(X)$, is the minimal $n$, for which $X$ can be covered by $n$ open categorical subsets. A standard reference is [5].

Remark 2.1 Contractible subsets of $X$ are clearly categorical, but the converse is not true-e.g., the sphere is a categorical subset of the ball. There is a related concept called geometric category, defined as the minimal cardinality of a cover of $X$ by open contractible sets (see [5, Chap. 3]). Like the strict covering type, the geometric 
category is not a homotopy invariant of $X$, so one defines the strong category, Cat $(X)$, as the minimum of geometric categories of spaces that are homotopy equivalent to $X$. Although the categorical sets may be very different from contractible ones, the following remarkable relation holds: $\operatorname{cat}(X) \leq \operatorname{Cat}(X) \leq \operatorname{cat}(X)+1$ (see [5, Prop. 3.15]).

Little is known about analogous relationships between the covering type and the strict covering type. For the wedge $W_{n}$ on $n$ circles Karoubi and Weibel [9, Prop. 4.1] show that $\operatorname{sct}\left(W_{n}\right)=n+2$, while $\operatorname{ct}\left(W_{n}\right)=\left\lceil\frac{3+\sqrt{1+8 n}}{2}\right\rceil$, which means that the difference between the two can be arbitrarily large. Furthermore, as we mentioned before, Borghini and Minian [3, Prop. 3.4] found an example of a closed surface for which the covering type is one less than its strict covering type.

The relation between the category and the covering type of a space is also complicated. For spheres cat $\left(S^{n}\right)=2$ while $\operatorname{ct}\left(S^{n}\right)=n+2$. Neither of them determines the other. We will give below examples of spaces that have the same covering type and yet the difference between respective categories is as big as we want. Nevertheless, if the category of a space is $n>1$, then its (homotopy) dimension is at least $n-1$ and so its covering type is at least $n+1$ (because it is not contractible). Roughly speaking, spaces with big category cannot have small covering type. We are going to make this statement more precise in the rest of this section.

Let us begin with a list of facts on which we will base the proofs of our results.

(A) By the Nerve theorem, if $X$ admits a good cover $\mathcal{U}$ of order $\leq n$ (i.e., at most $n$ different sets have non-empty intersection), then $X$ is homotopy equivalent to a simplicial complex of dimension $n-1$.

(B) By the Nerve theorem, if $U_{1}, \ldots, U_{n}$ are elements of a good cover that intersect non-trivially, then $U_{1} \cup \cdots \cup U_{n}$ is homotopy equivalent to $\Delta_{n-1}$, and therefore contractible.

(C) If cat $(X) \geq n, X=U \cup V$, where $U, V$ are open and $U$ is contractible (or more generally, $U$ is categorical in $X)$, then $\operatorname{cat}(V) \geq n-1$. This is obvious, because cat $(V)<n-1$ would imply cat $(U \cup V)<n$.

(D) $\operatorname{cat}(X) \leq \operatorname{hdim}(X)+1$, where $\operatorname{hdim}(X)$ is the homotopy dimension of $X$, defined as $\operatorname{hdim}(X):=\min \{\operatorname{dim}(Y) \mid Y \simeq X, Y$ is a CW-complex $\}$. The claim follows from the classical estimate $\operatorname{cat}(Y) \leq \operatorname{dim} Y+1$ and the homotopy invariance of LS-category.

\section{Theorem 2.2}

$$
\operatorname{ct}(X) \geq \frac{1}{2} \operatorname{cat}(X)(\operatorname{cat}(X)+1)
$$

Proof Assume that $X$ has a good cover $\mathcal{U}$ of cardinality $\operatorname{ct}(X)$. We proceed by induction. If $\operatorname{cat}(X)=1$ then $X$ is contractible, thus $\operatorname{ct}(X)=1$ and the inequality reduces to $1 \geq 1$.

Assume that the estimate holds for spaces with category $\leq n$, and let cat $(X)=n+1$. Then (D) implies $\operatorname{hdim}(X) \geq n$, so by (A) there exist sets $U_{1}, \ldots, U_{n+1} \in \mathcal{U}$ which intersect non-trivially. Let $U:=U_{1} \cup \cdots \cup U_{n+1}$ and let $V$ be the union of remaining 
elements of $\mathcal{U}$. Then $U$ is contractible by (B), which gives cat $(V) \geq n$ by $(\mathrm{C})$. We use the induction assumption to compute

$$
\operatorname{ct}(X) \geq(n+1)+\frac{1}{2} n(n+1)=\frac{1}{2}(n+1)(n+2) .
$$

Direct application of the theorem gives the following estimates. For spheres $\operatorname{cat}\left(S^{n}\right)=2$, therefore $\operatorname{ct}\left(S^{n}\right) \geq 3$, and for surfaces (with the exception of $S^{2}$ ) $\operatorname{cat}(P)=3$, therefore $\operatorname{ct}(P) \geq 6$. Furthermore, for real and complex projective $\operatorname{spaces} \operatorname{cat}\left(\mathbb{R} P^{n}\right)=\operatorname{cat}\left(\mathbb{C} P^{n}\right)=n+1$, so that $\operatorname{ct}\left(\mathbb{R} P^{n}\right) \geq(n+1)(n+2) / 2$ and $\operatorname{ct}\left(\mathbb{C} P^{n}\right) \geq(n+1)(n+2) / 2$.

A comparison with the results of [9] shows that some of the above estimates are not optimal and can be improved. In fact, we neglected the information about the dimension and connectivity of $X$, which also have an impact on the covering type. By taking these data into account we obtain much better estimates (except for real projective spaces, which are only 0 -connected and the category is directly related to the dimension). Nevertheless it is interesting to observe that the covering type increases (at least) quadratically with the category of the space.

\section{Theorem 2.3}

$$
\operatorname{ct}(X) \geq 1+\operatorname{hdim}(X)+\frac{1}{2} \operatorname{cat}(X)(\operatorname{cat}(X)-1) .
$$

Proof By fact (A) there exist sets $U_{1}, \ldots, U_{\mathrm{hdim}(X)+1} \in \mathcal{U}$ that intersect non-trivially. Let $U=U_{1} \cup \cdots \cup U_{\operatorname{hdim}(X)+1}$, and let $V$ be the union of remaining elements of $\mathcal{U}$. As above $\operatorname{cat}(V) \geq \operatorname{cat}(X)-1$, which together with Theorem 2.2 yields

$$
\operatorname{ct}(X) \geq(\operatorname{hdim}(X)+1)+\frac{1}{2} \operatorname{cat}(X)(\operatorname{cat}(X)-1) .
$$

A similar approach can be used to estimate the minimal number of points (vertices) that are required in order to triangulate a given PL-manifold. Recall that a triangulation of a manifold is combinatorial if the links of all vertices are triangulated spheres. Then we have the following

Corollary 2.4 Let $M$ be a d-dimensional and c-connected closed PL-manifold. Then

$$
\Delta^{\mathrm{PL}}(M) \geq 1+d+c \cdot(\operatorname{cat}(M)-2)+\frac{1}{2} \operatorname{cat}(M)(\operatorname{cat}(M)-1) .
$$

Proof Note that the case $c=0$ is covered by Theorem 2.3. Similarly, the claim is correct if we assume $c=d-1$, because that implies $M \simeq S^{d}$ and $\operatorname{ct}\left(S^{d}\right)=d+2$. Finally, if $d=2$ and $M$ is 0 -connected but not 1-connected, then $M$ is a closed surface other than sphere, hence cat $(M)=3$, and the above formula claims that $\Delta^{\mathrm{PL}}(M) \geq 6$, which we already know. Therefore we may assume from this point on that $d \geq 3$ and $1 \leq c \leq d-2$. 
Let $K$ be a combinatorial triangulation of $M$ and let $\mathcal{U}$ be the good cover of $M$ formed by open stars of vertices of $K$. Since $M$ is $d$-dimensional, there are at least $d+1$ open stars that intersect non-trivially. Their union $U$ is the simplicial neighbourhood of a $d$-dimensional simplex, and the intersection of $U$ with the union $V$ of all other open stars is homeomorphic to $S^{d-1} \times(0,1)$.

Since $d \geq 3$, the Seifert-van Kampen theorem implies that $V$ is simply connected. Furthermore, since $c \leq d-2$ the Mayer-Vietoris sequence for the cover $\{U, V\}$ of $M$ shows that $H_{i}(V) \cong H_{i}(M)$ for $i \leq c$, thus $V$ is also $c$-connected.

We have seen previously that $\operatorname{cat}(V) \geq \operatorname{cat}(M)-1$, so we use the known inequality (see [5])

$$
\operatorname{cat}(V) \leq \frac{\operatorname{hdim}(V)}{c+1}+1
$$

to deduce that $\operatorname{hdim}(V) \geq(c+1)(\operatorname{cat}(M)-2)$. As before, this implies that at least $(c+1)(\operatorname{cat}(M)-2)+1$ of the open stars that cover $V$ have a non-trivial intersection, and that $W$, the union of the remaining open stars has category cat $(W) \geq \operatorname{cat}(M)-2$. By applying Theorems 1.2 and 2.3 to estimate the number of vertices in $W$ we may conclude that $K$ has at least

$$
\begin{aligned}
1 & +d+(c+1)(\operatorname{cat}(M)-2)+1+\frac{1}{2}(\operatorname{cat}(M)-1)(\operatorname{cat}(M)-2) \\
& =1+d+c \cdot(\operatorname{cat}(M)-2)+\frac{1}{2} \operatorname{cat}(M)(\operatorname{cat}(M)-1)
\end{aligned}
$$

vertices.

Observe that this estimate is a strict improvement of Theorem 2.3 for all PLmanifolds which are at least 1-connected and are not spheres. For example, it shows that every triangulation of $\mathbb{C} P^{n}$ requires at least $n(n+7) / 2$ vertices.

We may also reverse the above estimates to obtain upper bounds for the category of a space based on the cardinality of good cover or the number of vertices in a triangulation.

Corollary 2.5 Assume that $X$ admits a good cover with $n$ elements. Then the category of $X$ is bounded above by

$$
\operatorname{cat}(X) \leq \frac{-1+\sqrt{1+8 n}}{2}
$$

If the dimension of $X$ is known we have also a better estimate

$$
\operatorname{cat}(X) \leq \frac{1+\sqrt{1+8(n-\operatorname{hdim}(X)-1)}}{2} .
$$

Proof The estimates are easily proved by solving the inequalities in Theorems 2.2 and 2.3 for $\operatorname{cat}(X)$.

We mentioned the general estimate $\operatorname{ct}(X)-\operatorname{hdim}(X) \geq 2$ which holds for every non-contractible space $X$. By using the above inequalities we easily see that $\operatorname{ct}(X)-$ 
$\operatorname{hdim}(X) \leq 3$ implies cat $(X) \leq 2$ and that $\operatorname{ct}(X)-\operatorname{hdim}(X) \leq 6 \operatorname{implies} \operatorname{cat}(X) \leq 3$. This leads to the following interesting result.

Corollary 2.6 Assume that $\operatorname{ct}(X)-\operatorname{hdim}(X) \leq 3$ or that $X$ is a closed manifold (of dimension at least 3$)$ and $\operatorname{ct}(X)-\operatorname{dim}(X) \leq 6$. Then the fundamental group of $X$ is free.

Proof If $\operatorname{ct}(X)-\operatorname{hdim}(X) \leq 3$ then by Corollary 2.5 the category of $X$ is at most 2, and a well-known result (see [5, Section 1.6]) implies that $\pi_{1}(X)$ is a free group.

Similarly, if $\operatorname{ct}(X)-\operatorname{hdim}(X) \leq 6$, then the category of $X$ is at most 3. Our claim then follows from the main result of [7] that the fundamental group of a closed manifold whose fundamental group is not free must have category at least 4 . Note that the second statement holds for closed surfaces as well, with the exception of the torus, the projective plane and the Klein bottle.

\section{Cohomological Estimates}

It is well known that the Lusternik-Schnirelmann category of a space $X$ is closely related to the structure of the cohomology ring $\widetilde{H}^{*}(X)$. Indeed, cat $(X)$ is bounded below by the so-called cup-length of $X$, which is defined as the maximal number of factors among all non-trivial products in $\widetilde{H}^{*}(X)$ (and with any coefficients, see [5, Prop. 1.5]). However, that estimate does not involve the respective dimensions of the factors in the product. We are going to show that the latter play an important role in the estimate of covering type, which will lead to considerable improvements in our estimates of the covering type of $X$.

Given an $n$-tuple of positive integers $i_{1}, \ldots, i_{n} \in \mathbb{N}$ we will say that a space $X$ admits an essential $\left(i_{1}, \ldots, i_{n}\right)$-product if there are cohomology classes $x_{k} \in H^{i_{k}}(X)$, such that the product $x_{1} x_{2} \cdots x_{n}$ is non-trivial. For every $\left(i_{1}, \ldots, i_{n}\right)$ there exists a space $X$ that admits an essential $\left(i_{1}, \ldots, i_{n}\right)$-product, for example we can take $X=S^{i_{1}} \times \cdots \times S^{i_{n}}$. Clearly, if $X$ admits an essential $\left(i_{1}, \ldots, i_{n}\right)$-product then so does every $Y \simeq X$, since their cohomology rings are isomorphic. We may therefore define the covering type of the $n$-tuple of positive integers $\left(i_{1}, \ldots, i_{n}\right)$ as

$$
\operatorname{ct}\left(i_{1}, \ldots, i_{n}\right):=\min \left\{\operatorname{ct}(X) \mid X \text { admits an essential }\left(i_{1}, \ldots, i_{n}\right) \text {-product }\right\}
$$

The following proposition is a direct consequence of the definition.

\section{Proposition 3.1}

$$
\operatorname{ct}(X) \geq \max \left\{\operatorname{ct}\left(\left|x_{1}\right|, \ldots,\left|x_{n}\right|\right) \mid \text { for all } 0 \neq x_{1} \cdots x_{n} \in H^{*}(X)\right\}
$$

Although the covering type of a specific product of cohomology classes may appear as a coarse estimate, it will serve very well our purposes. We will base our computations on the following technical lemmas. The first is a standard argument that we give here for the convenience of the reader. 
Lemma 3.2 Let $X=U \cup V$ where $U, V$ are open in $X$, and let $x, y \in \widetilde{H}^{*}(X)$ be cohomology classes whose product $x \cdot y$ is non-trivial. If $U$ is categorical in $X$ then $i_{V}^{*}(x)$ is a non-trivial element of $H^{*}(V)$ (here $i_{V}$ stands for the inclusion map $\left.i_{V}: V \hookrightarrow X\right)$.

Proof Assume by contradiction that $i_{V}^{*}(x)=0$. Exactness of the cohomology sequence

$$
H^{*}(X, V) \stackrel{j_{V}^{*}}{\longrightarrow} H^{*}(X) \stackrel{i_{V}^{*}}{\longrightarrow} H^{*}(V)
$$

implies that there is a class $\bar{x} \in H^{*}(X, V)$ such that $j_{V}^{*}(\bar{x})=x$. Moreover $i_{U}^{*}(y)=0$, because $i_{U}: U \hookrightarrow X$ is null-homotopic, so there is a class $\bar{y} \in H^{*}(X, U)$ such that $j_{U}^{*}(\bar{y})=y$. Then $x \cdot y=j_{V}^{*}(\bar{x}) \cdot j_{U}^{*}(\bar{y})$ is by naturality equal to the image of $\bar{x} \cdot \bar{y} \in H^{*}(X, U \cup V)=0$, therefore $x \cdot y=0$, which contradicts the assumptions of the lemma.

By inductive application of the above lemma we obtain the following:

Lemma 3.3 Let $x_{1}, \ldots, x_{n} \in \widetilde{H}^{*}(X)$ be cohomology classes whose product $x_{1} \cdots x_{n}$ is non-trivial, and let $X=U_{1} \cup \cdots \cup U_{k} \cup V$ where $U_{1}, \ldots, U_{k}$ are open categorical subsets of $X$, and $V$ is open in $X$. Then the product of any $n-k$ different classes among $i_{V}^{*}\left(x_{1}\right), \ldots, i_{V}^{*}\left(x_{n}\right)$ is a non-trivial class in $H^{*}(V)$.

The following simple extension of [9, Prop. 3.1] will allow a slightly better estimate of $\operatorname{ct}\left(i_{1}, \ldots, i_{n}\right)$ if the parameters $i_{1}, \ldots, i_{n}$ are not all equal.

Lemma 3.4 If $X$ has non-trivial reduced homology groups in different dimensions, then $\operatorname{ct}(X) \geq \operatorname{hdim}(X)+3$.

Proof If $\operatorname{ct}(X) \leq \operatorname{hdim}(X)+2$, then $X$ is homotopy equivalent to a subcomplex of $\Delta_{\text {hdim }(X)+1}$. The only subcomplex of $\Delta_{\operatorname{hdim}(X)+1}$ that has homotopy dimension equal to $\operatorname{hdim}(X)$ is $\partial \Delta_{\operatorname{hdim}(X)+1}$, which has only one non-trivial reduced homology group.

We are ready to prove the main result of this section, an 'arithmetic' estimate for the covering type of an $n$-tuple.

\section{Theorem 3.5}

$$
\operatorname{ct}\left(i_{1}, \ldots, i_{n}\right) \geq i_{1}+2 i_{2}+\cdots+n i_{n}+(n+1) .
$$

If $i_{1}, \ldots, i_{n}$ are not all equal, then

$$
\operatorname{ct}\left(i_{1}, \ldots, i_{n}\right) \geq i_{1}+2 i_{2}+\cdots+n i_{n}+(n+2) .
$$

Proof The first statement can be proved by induction. Unfortunately, the same approach is not sufficient to prove the stronger statement, and a modified inductive argument turns out to be quite complicated, and we find it easier to give a direct proof. 
Although the second proof covers the first statement as well, we believe that it is still of some interest to be able to compare the two methods.

Toward the proof of the first statement, we begin the induction by observing that if $0 \neq x_{1} \in H^{i_{1}}(X)$ then $\operatorname{hdim}(X) \geq i_{1}$, hence $\operatorname{ct}\left(i_{1}\right) \geq i_{1}+2$ by [9, Prop. 3.1].

Assume that the estimate holds for all sequences of $n-1$ positive integers and consider the classes $x_{1} \in \widetilde{H}^{i_{1}}(X), \ldots, x_{n} \in \widetilde{H}^{i_{n}}(X)$ such that the product $x_{1} \cdots x_{n} \in$ $H^{i_{1}+\ldots+i_{n}}(X)$ is non-trivial. The cohomological dimension of $X$ is at least $i_{1}+\cdots+i_{n}$, therefore in every good cover $\mathcal{U}$ of $X$ one can find $i_{1}+\cdots+i_{n}+1$ elements that intersect non-trivially. Denote their union by $U$ and the union of the remaining elements of $\mathcal{U}$ by $V$. Then $U$ is contractible and by Lemma 3.3 there exists in $\widetilde{H}^{*}(V)$ a non-zero product of elements whose degrees are $i_{2}, \ldots, i_{n}$. By induction we obtain

$$
\begin{aligned}
\operatorname{ct}(X) & \geq\left(i_{1}+\cdots+i_{n}+1\right)+\left(i_{2}+2 i_{3}+\cdots+(n-1) i_{n}+n\right) \\
& =i_{1}+2 i_{2}+\cdots+n i_{n}+(n+1),
\end{aligned}
$$

which proves the first statement.

For the second statement, let $\mathcal{U}$ be a good cover of $X$, and assume that the product of classes $x_{1} \in \widetilde{H}^{i_{1}}(X), \ldots, x_{n} \in \widetilde{H}^{i_{n}}(X)$ is non-trivial. As before, there exists $\mathcal{U}_{1} \subseteq \mathcal{U}$, such that $\mathcal{U}_{1}$ contains $i_{1}+\cdots+i_{n}+1$ sets that intersect non-trivially. If we denote by $V_{1}$ the union of sets in $\mathcal{U}-\mathcal{U}_{1}$, then by Lemma 3.3 the restriction to $V_{1}$ of any sub-product of $x_{1} \cdots x_{n}$ of length $n-1$ is non-trivial. In particular, $H^{i_{2}+\cdots+i_{n}}\left(V_{1}\right) \neq 0$, and so there exists $\mathcal{U}_{2} \subseteq \mathcal{U}-\mathcal{U}_{1}$ such that $\mathcal{U}_{2}$ contains $i_{2}+\cdots+i_{n}+1$ sets that intersect non-trivially. By continuing this procedure we end up with disjoint collections $\mathcal{U}_{1}, \ldots, \mathcal{U}_{n-1} \subseteq \mathcal{U}$, where each $\mathcal{U}_{k}$ has $i_{k}+\cdots+i_{n}+1$ elements and the union of its elements is contractible.

Let $V$ denote the union of all elements in $\mathcal{U}_{n}:=\mathcal{U}-\mathcal{U}_{1}-\cdots-\mathcal{U}_{n-1}$. By Lemma 3.3, $H^{*}(V)$ has non-trivial cohomology classes in dimensions $i_{1}, \ldots, i_{n}$. Since we assumed that they are not all equal, Lemma 3.4 implies that $\mathcal{U}_{n}$ has at least $i_{n}+3$ elements. By adding up the cardinalities of all $\mathcal{U}_{k}$ we conclude that $\mathcal{U}$ has at least $i_{1}+2 i_{2}+\cdots+n i_{n}+(n+2)$ elements.

It is worth to emphasise that it is usually not difficult to identify the cup product in $\widetilde{H}^{*}(X)$ which provides the best estimate for the covering type. In particular, it clearly makes sense to consider only products whose terms have non-decreasing degrees. The rest of the section is dedicated to computations of specific examples (projective spaces, products of spheres, etc.) based on Theorem 3.5.

Corollary 3.6 The covering type of projective spaces is bounded by $\operatorname{ct}\left(\mathbb{R} P^{n}\right) \geq \frac{1}{2}(n+1)(n+2), \quad \operatorname{ct}\left(\mathbb{C} P^{n}\right) \geq(n+1)^{2}, \quad \operatorname{ct}\left(\mathbb{H} P^{n}\right) \geq(n+1)(2 n+1)$

Proof By Theorem 3.5 it is sufficient to estimate

$$
\operatorname{ct}(\underbrace{k, \ldots, k}_{n}) \geq k+2 k+\cdots+n k+n+1=\frac{1}{2}(n+1)(k n+2),
$$


because the real, complex and quaternionic projective spaces correspond respectively to cases $k=1,2,4$.

For a product of spheres $X=S^{i_{1}} \times \cdots \times S^{i_{n}}$ where $i_{1} \leq \cdots \leq i_{n}$ are not all equal, Theorem 3.5 yields $\operatorname{ct}(X) \geq i_{1}+2 i_{2}+\cdots+n i_{n}+(n+2)$, while for a product of spheres of same dimension we get

$$
\operatorname{ct}\left(\left(S^{i}\right)^{n}\right) \geq \frac{(n+1)(n i+2)}{2} .
$$

The last estimate can be sometimes improved by ad hoc methods, see Example 3.9.

Corollary 3.7 The covering type of unitary groups is estimated as

$$
\operatorname{ct}(U(n)) \geq \frac{1}{6}\left(4 n^{3}+3 n^{2}+5 n+12\right) \text { and } \operatorname{ct}(S U(n)) \geq \frac{1}{6}\left(4 n^{3}-3 n^{2}+5 n+6\right) .
$$

Proof The cohomology algebra $H^{*}(U(n))$ is the exterior algebra on generators in dimensions $1,3, \ldots, 2 n-1$, while $H^{*}(S U(n))$ is the exterior algebra on generators in dimensions $3,5, \ldots, 2 n-1$. Theorem 3.5 gives

$$
\begin{aligned}
\operatorname{ct}(U(n)) & \geq \operatorname{ct}(1,3, \ldots, 2 n-1) \geq 1+2 \cdot 3+3 \cdot 5+\cdots+n \cdot(2 n-1)+(n+2) \\
& =\frac{1}{6}\left(4 n^{3}+3 n^{2}+5 n+12\right)
\end{aligned}
$$

and

$$
\begin{aligned}
\operatorname{ct}(S U(n)) & \geq \operatorname{ct}(3,5, \ldots, 2 n-1) \\
& \geq 1 \cdot 3+2 \cdot 5+\cdots+(n-1) \cdot(2 n+1)+(n+1) \\
& =\frac{1}{6}\left(4 n^{3}-3 n^{2}+5 n+6\right) .
\end{aligned}
$$

The LS-category of unitary groups is $\operatorname{cat}(U(n))=n$ and $\operatorname{cat}(S U(n))=n-1$ (see [5, Thm. 9.47]), so our estimate of the covering type is a cubical function of the category (as compared with results from Sect. 2 where we obtained a general quadratic relation between the category and the covering type).

Remark 3.8 The estimates of Corollary 3.6 applied to the number of vertices of triangulation of $\mathbb{R} P^{n}$ and $\mathbb{C} P^{n}$ or spaces with the same cohomology algebra reprove the result of [1]. The corresponding estimate for $\mathbb{H} P^{n}$ has not been stated in the literature, up to our knowledge.

The estimate of number of vertices in a triangulation of $U(n)$, or $S U(n)$, that follows from our estimate of the covering type in Corollary 3.7 is new.

The computation for unitary groups can be easily extended to finite, (homotopy) associative $H$-spaces, i.e., spaces with a continuous product that is associative only 
up to a suitable homotopy (see [11, Sect. III, 4]). In fact the $\mathbb{Z}_{p}$-cohomology of a finite associative $H$-space is given as (see [11, Thm. III, 8.7])

$$
H^{*}\left(X ; \mathbb{Z}_{p}\right) \cong \bigotimes_{i=1}^{n} \mathbb{Z}_{p}\left[x_{i}\right] /\left(x_{i}^{k_{i}}\right)
$$

where $k_{i}$ is a power of 2 if $p=2$, while for $p$ odd there are two cases: $k_{i}=2$ if $\left|x_{i}\right|$ is odd, and $k_{i}$ is a power of $p$ if $\left|x_{i}\right|$ is even. Thus, for a given prime $p$ we may consider the corresponding structure of $H^{*}\left(X ; \mathbb{Z}_{p}\right)$ and define

$$
\operatorname{ct}_{p}(X):=\operatorname{ct}(\underbrace{\left|x_{1}\right|, \ldots,\left|x_{1}\right|}_{k_{1}-1}, \ldots, \underbrace{\left|x_{n}\right|, \ldots,\left|x_{n}\right|}_{k_{n}-1}) .
$$

A specific $H$-space may have a trivial cohomology structure with respect to some coefficient fields and very rich with respect to other fields, yielding different values of $\mathrm{ct}_{p}(X)$. The crucial observation is that the covering type yields a uniform bound for all of them so we have the following inequality:

$$
\operatorname{ct}(X) \geq \max _{p \text { prime }}\left\{\operatorname{ct}_{p}(X)\right\} .
$$

In particular, we can easily compute lower estimates for the covering type of all classical Lie groups, since their cohomology rings are well known.

Let us mention that for spaces whose cohomology algebra has several linearly independent generators in low dimensions it is possible to improve the general estimates of the covering type. Since the actual improvements arise only in few cases, we do not attempt to develop a theory but instead illustrate this method on an example.

Example 3.9 We are going to estimate $\operatorname{ct}\left(S^{1} \times S^{1} \times S^{1}\right)$. Let $\mathcal{U}$ be a good cover of some $X$ that is homotopy equivalent to $S^{1} \times S^{1} \times S^{1}$. Then with respect to any field coefficients we have $H^{*}(X) \cong \Lambda(x, y, z)$, where $|x|=|y|=|z|=1$. Since $\operatorname{hdim}(X)=3$, there are at least four open sets, say $U_{1}, U_{2}, U_{3}, U_{4} \in \mathcal{U}$ that intersect non-trivially. The union of the remaining elements of $\mathcal{U}$ has category at least three and hdim at least two. By Theorem 2.3 a good cover of it has at least six sets, so there are also sets $U_{5}, \ldots, U_{10} \in \mathcal{U}$. Observe that by the Nerve theorem $H^{1}\left(U_{5} \cup U_{6} \cup U_{7}\right)$ is at most 1-dimensional, and similarly for $H^{1}\left(U_{8} \cup U_{9} \cup U_{10}\right)$. Since $H^{1}(X)$ is 3-dimensional, the kernel of the restriction homomorphism

$$
H^{1}(X) \rightarrow H^{1}\left(U_{5} \cup U_{6} \cup U_{7}\right) \oplus H^{1}\left(U_{8} \cup U_{9} \cup U_{10}\right)
$$

contains a non-trivial element $u \in H^{1}(X)$. Moreover, the kernel of the restriction homomorphism

$$
H^{1}(X) \rightarrow H^{1}\left(U_{5} \cup U_{6} \cup U_{7}\right)
$$


is at least 2-dimensional so we may find in it a non-trivial element $v \in H^{1}(X)$ which is linearly independent from $u$. Finally, we can choose $w \in H^{1}(X)$ so that the set $\{u, v, w\}$ is a basis of $H^{1}(X)$.

The restriction of $u$ to $U_{8} \cup U_{9} \cup U_{10}$ is trivial, so by exactness of the cohomology sequence of the pair there exists

$$
\bar{u} \in H^{1}\left(X, U_{8} \cup U_{9} \cup U_{10}\right)
$$

such that $j^{*}(\bar{u})=u$ (where $j^{*}$ denotes the homomorphism induced by the inclusion of $X$ in the pair). Similarly, one can find

$$
\bar{v} \in H^{1}\left(X, U_{5} \cup U_{6} \cup U_{7}\right), \quad \bar{w} \in H^{1}\left(X, U_{1} \cup \cdots \cup U_{4}\right)
$$

satisfying $j^{*}(\bar{v})=v$ and $j^{*}(\bar{w})=w$. Since $0 \neq u \cdot v \cdot w=j^{*}(\bar{u} \cdot \bar{v} \cdot \bar{w})$ and $\bar{u} \cdot \bar{v} \cdot \bar{w} \in H^{3}\left(X, U_{1} \cup \cdots \cup U_{10}\right)$, we conclude that $X \neq U_{1} \cup U_{2} \cdots \cup U_{10}$, therefore $\operatorname{ct}(X) \geq 11$.

Note that it is an improvement of estimate of Theorem 3.5 which gives

$$
\operatorname{ct}(X) \geq \operatorname{ct}(1,1,1) \geq 1+2+3+4=10 .
$$

It is worth pointing out that for the product of four circles the two methods give the same estimate $\operatorname{ct}\left(S^{1} \times S^{1} \times S^{1} \times S^{1}\right) \geq 15$, while for more than 4 generators Theorem 3.5 yields a better estimate of the covering type.

\section{Moore Spaces}

In this section we estimate the covering type of various Moore spaces and use the results to derive estimates for related spaces. Recall that for every abelian group $A$ and positive integer $i$ one can construct a CW complex $X$ with

$$
\widetilde{H}_{k}(X)= \begin{cases}A, & k=i, \\ 0, & k \neq i\end{cases}
$$

Any such space is called a Moore space of type $M(A, i)$ (cf. [8, Exam. 2.40]). If $i>1$ we normally assume that $X$ is simply connected, because then $A$ and $i$ uniquely determine the homotopy type of $X$ (see [8, Exam. 3.34]), and we may write $M(A, i)$ instead of $X$. Homotopy uniqueness fails if $i=1$ so we normally consider specific constructions of $M(A, 1)$ (see below). The simplest examples are wedges of spheres: $r$-fold wedge of $i$-dimensional spheres is a Moore space of type $M\left(\mathbb{Z}^{r}, i\right)$.

By Theorem 1.2 every space $X$ with $\operatorname{ct}(X)=n$ is homotopy equivalent to a subcomplex of $\Delta_{n-1}$. Therefore, for any given $n$ there exist only finitely many homotopy types of spaces whose covering type is equal to $n$, and we may even attempt a classification, at least for small values of $n$. For each $n$ there is always a trivial example of a space with $\operatorname{ct}(X)=n$, namely the discrete space with $n$ points. These are the only 
spaces with covering type 1 or 2 . The first non-trivial example is the circle, whose covering type is 3 , and belongs to the family of spheres $S^{n}$ whose covering type is $\operatorname{ct}\left(S^{n}\right)=n+2$. Apart from the discrete space and the sphere, there are two other spaces with covering type 4 , namely the wedges of two and of three circles. Similarly, the spaces of covering type 5 are wedges of spheres of various dimensions. The number of homotopy distinct complexes increases rapidly with the covering type, but there is a reasonably complete classification for manifolds whose covering type is at most 11 (cf. [6, Sect. 5]).

The following theorem gives the covering type of Moore spaces with free homology.

Theorem $4.1 \operatorname{ct}\left(M\left(\mathbb{Z}^{r}, i\right)\right)=n$, where $n$ is the minimal integer for which $\left(\begin{array}{c}n-1 \\ i+1\end{array}\right) \geq r$.

Proof The case $i=1$ is covered by [9, Prop. 4.1], so we may assume $i>1$, and thus avoid complications with the fundamental group.

Let us first compute the homology of $\Delta_{n-1}^{(i)}$, the $i$-th skeleton of the $(n-1)$ dimensional simplex. The simplicial chain complex of $\Delta_{n-1}^{(i)}$ is obtained by truncating the simplicial chain complex for $\Delta_{n-1}$ at degree $i$ :

$$
C_{i}\left(\Delta_{n-1}\right) \stackrel{\partial_{i}}{\longrightarrow} C_{i-1}\left(\Delta_{n-1}\right) \longrightarrow \cdots \longrightarrow C_{0}\left(\Delta_{n-1}\right) \stackrel{\partial_{0}}{\longrightarrow} C_{-1}=\mathbb{Z} .
$$

The homology of $\Delta_{n-1}$ is trivial, so the above chain complex is exact, except at the beginning. The rank of each $C_{k}\left(\Delta_{n-1}\right)$ is $\left(\begin{array}{c}n \\ k+1\end{array}\right)$, and the rank of $H_{i}\left(\Delta_{n-1}^{(i)}\right)=\operatorname{ker} \partial_{i}$ can be computed by exploiting the exactness:

$$
\operatorname{rank}\left(\operatorname{ker} \partial_{i}\right)=\left(\begin{array}{c}
n \\
i+1
\end{array}\right)-\left(\begin{array}{c}
n \\
i
\end{array}\right)+\cdots+(-1)^{i}\left(\begin{array}{c}
n \\
1
\end{array}\right)+(-1)^{i+1}\left(\begin{array}{c}
n \\
0
\end{array}\right)=\left(\begin{array}{c}
n-1 \\
i+1
\end{array}\right)
$$

Since $\Delta_{n-1}^{(i)}$ is clearly simply connected by the standard computation of the fundamental group of a simplicial complex, we conclude that it is a Moore space of type $M\left(\mathbb{Z}^{\left(\begin{array}{c}n-1 \\ i+1\end{array}\right)}, i\right)$.

It is obvious from the definition of simplicial homology that the rank of $H_{i}\left(\Delta_{n-1}^{(i)}\right)$ is maximal among all sub-complexes of $\Delta_{n-1}$. Therefore, if $r>\left(\begin{array}{c}n-1 \\ i+1\end{array}\right)$, then $M\left(\mathbb{Z}^{r}, i\right)$ cannot be represented by a subcomplex of $\Delta_{n-1}$.

To show the converse, note that $\operatorname{im}\left(\partial_{i}\right)$ is $\left(\begin{array}{c}n-2 \\ i+1\end{array}\right)$-dimensional, so we may find up to $\left(\begin{array}{c}n \\ i+1\end{array}\right)-\left(\begin{array}{c}n-2 \\ i+1\end{array}\right)=\left(\begin{array}{c}n-1 \\ i+1\end{array}\right) i$-simplices in $\Delta_{n-1}^{(i)}$ whose removal does not alter the image of $\partial_{i}$. In particular, if $r \leq\left(\begin{array}{c}n-1 \\ i+1\end{array}\right)$ then we may remove $\left(\begin{array}{c}n-1 \\ i+1\end{array}\right)-r$ simplices of dimension $i$, so that the remaining simplices form a Moore space of type $M\left(\mathbb{Z}^{r}, i\right)$. We conclude that $M\left(\mathbb{Z}^{r}, i\right)$ can be represented by a subcomplex of $\Delta_{n-1}$ if and only if $\left(\begin{array}{c}n-1 \\ i+1\end{array}\right) \geq r$, which proves our claim.

The theorem that we have just proved allows to improve some of our previous estimates. Let $M$ be an $(n-1)$-connected closed $2 n$-dimensional manifold. Up to homotopy type it can be built by attaching a $2 n$-dimensional sphere to a wedge of $n$-dimensional spheres. Its LS-category is 3 , so by Corollary 2.4 every combinatorial triangulation of $M$ has at least 


$$
1+2 n+(n-1)+\frac{1}{2} \cdot 3 \cdot 2=3 n+3
$$

vertices. Similarly, Poincaré duality implies that there are cohomology classes in $H^{n}(M)$ whose product is non zero, so by Proposition 3.1 and Theorem 3.5 the covering type of $M$ is bounded by

$$
\operatorname{ct}(M) \geq \operatorname{ct}(n, n)=3 n+3 .
$$

We can obtain better estimates by taking into account the rank of $H_{n}(M)$.

Corollary 4.2 Let M be an (n-1)-connected and $2 n$-dimensional closed PL-manifold. Then every combinatorial triangulation of $M$ has at least $3 n+3+k$ vertices, where $k$ is the minimal integer for which $\left(\begin{array}{c}n+k+1 \\ k\end{array}\right) \geq \operatorname{rank} H_{n}(M)$.

Proof The first part of the argument is similar as in the proof of Corollary 2.4. Let $K$ be a combinatorial triangulation of $M$ and let $\mathcal{U}$ be the cover of $M$ by open stars of vertices in $K$. Then there exist $2 n+1$ vertices in $K$ that span a $2 n$-dimensional simplex, and the union $U$ of corresponding open stars form the simplicial neighbourhood of that simplex. If we denote by $V$ the union of the remaining open stars, then the intersection $U \cap V$ is homeomorphic to $S^{2 n-1} \times(0,1)$.

If $n>1$ then the exactness of the homology sequence of the pair $(M, V)$ immediately implies that $V$ is a Moore space of type $M\left(\mathbb{Z}^{r}, n\right)$ where $r=\operatorname{rank}\left(H_{n}(M)\right)$. If $n=1$, then we observe that $V$ can be deformed to a surface with boundary, and these are well known to be homotopy equivalent to wedges of circles. In that case $V$ is a Moore space of type $M\left(\mathbb{Z}^{r}, 1\right)$ where $r=\operatorname{rank}_{\mathbb{Z}_{2}}\left(H_{1}\left(M ; \mathbb{Z}_{2}\right)\right.$ ) (we use $\mathbb{Z}_{2}$-coefficients to obtain a statement that is valid for both orientable and non-orientable surfaces).

By Theorem 4.1, $V$ is the union of at least $n+k+2$ open stars of vertices in $K$, where $k$ is the minimal integer for which $\left(\begin{array}{c}n+k+1 \\ k\end{array}\right)=\left(\begin{array}{c}n+k+1 \\ n+1\end{array}\right) \geq r$. We conclude that $K$ has at least $(2 n+1)+(n+k+2)=3 n+3+k$ vertices, where $k$ is defined as above.

Corollary 4.3 Let $m \leq n$. Then we have

$$
\operatorname{ct}\left(S^{m} \times S^{n}\right) \geq m+2 n+4
$$

Proof For $m<n$ the estimate follows by Theorem 3.5, and for $m=n$ by the previous corollary and the observation that $k=n+2$ is the minimal integer for which $\left(\begin{array}{c}k \\ n+1\end{array}\right) \geq$ rank $H_{n}\left(S^{n} \times S^{n}\right)=2$.

We must add that the corresponding estimate of number of vertices of a triangulation of a combinatorial manifold which is homotopy equivalent to $S^{m} \times S^{n}$ which follows from Corollary 4.3 was shown in [4] (see also [2]).

Moore spaces for an arbitrary abelian group are usually constructed as wedges of Moore spaces of cyclic groups. It is therefore important to have estimates of the covering type of a wedge of spaces but one should expect some irregular behaviour. For example, by [9, Prop. 4.1] we have the following relations:

$$
\operatorname{ct}\left(S^{1}\right)<\operatorname{ct}\left(S^{1} \vee S^{1}\right)=\operatorname{ct}\left(S^{1} \vee S^{1} \vee S^{1}\right)<\operatorname{ct}\left(S^{1} \vee S^{1} \vee S^{1} \vee S^{1}\right)
$$


We can derive an upper estimate for the covering type of a wedge as follows. Let $m=\operatorname{ct}(X)$ and $n=\operatorname{ct}(Y)$. Then there are simplicial complexes $K \leq \Delta_{m-1}$ and $L \leq \Delta_{n-1}$, such that $X \simeq|K|$ and $Y \simeq|L|$. Clearly, $K \vee L$ can be realised as anepoint union of $K$ and $L$ and is thus a subcomplex of $\Delta_{m+n-2}$. That estimate can be improved by gluing $K$ and $L$ along bigger subcomplexes. Indeed, if hdim(X) $=k$ and $\operatorname{hdim}(Y)=l$, then $K$ and $L$ contain respectively a $k$-dimensional simplex $\sigma \leq K$ and an $l$-dimensional simplex $\tau \leq L$. If we assume that $k \leq l$ and we glue together $K$ and $L$ so that $\sigma$ is identified with a face of $\tau$, then the resulting complex is a subcomplex of $\Delta_{m+n-k-2}$, while its geometric realisation is homotopy equivalent to $X \vee Y$. Thus we have proved the following estimate:

\section{Proposition 4.4}

$$
\operatorname{ct}(X \vee Y) \leq \operatorname{ct}(X)+\operatorname{ct}(Y)-\min \{\operatorname{hdim} X, \operatorname{hdim} Y\}-1
$$

Karoubi and Weibel [9, Thm. 7.1] have shown that the suspension of a space can increase its covering type at most by one. However, it happens frequently that the covering type drops after suspension.

Example 4.5 We have shown in Corollary 4.3 that $\operatorname{ct}\left(S^{m} \times S^{n}\right) \geq m+2 n+4$. On the other hand, after suspension a product of spheres splits as a wedge of spheres $\Sigma\left(S^{m} \times S^{n}\right) \simeq S^{m+1} \vee S^{n+1} \vee S^{m+n+1}$. Therefore, by Proposition 4.4 $\operatorname{ct}\left(\Sigma\left(S^{n} \times\right.\right.$ $\left.\left.S^{n}\right)\right) \leq m+n+5$, so the covering type of the suspension of $S^{m} \times S^{n}$ is smaller at least by $n-1$ than the covering type of $S^{m} \times S^{n}$. Indeed, the result is not surprising at all if we have in mind our estimates based on the LS-category and the cohomology products, and recall that the category of a suspension is always equal to 2 , and that the cohomology products in a suspension are always trivial.

Recall that we may construct Moore space whose homology is a finite cyclic group as follows. Let $X$ be the 2-dimensional CW complex obtained by attaching a 2-cell to a circle by a map of degree $k$. The computation of cellular homology shows that $X$ is a Moore space of type $M\left(\mathbb{Z}_{k}, 1\right)$. Moreover, the $(i-1)$-fold suspension of $X$ is clearly a Moore space of type $M\left(\mathbb{Z}_{k}, i\right)$. To avoid repetition in the statements of the following two results we adopt the convention that $M(A, 1)$ denotes the specific construction (wedge of circles and spaces of type $M\left(\mathbb{Z}_{k}, 1\right)$ that we have just described).

\section{Proposition 4.6}

$$
i+3 \leq \operatorname{ct}\left(M\left(\mathbb{Z}_{k}, i\right)\right) \leq i+3 k
$$

Proof The lower estimate follows immediately from $\operatorname{hdim} M\left(\mathbb{Z}_{k}, i\right)=i+1$. For the upper estimate recall that $M\left(\mathbb{Z}_{k}, 1\right)$ is the mapping cone of a degree $k$ map between circles. By [9, Thm. 7.2] and the fact that $\operatorname{ct}\left(S^{1}\right)=3$ we obtain $\operatorname{ct}\left(M\left(\mathbb{Z}_{k}, 1\right)\right) \leq 1+3 k$. Since $M\left(\mathbb{Z}_{k}, i\right)$ can be obtained as an $(i-1)$-fold suspension of $M\left(\mathbb{Z}_{k}, 1\right)$, [9, Thm. 7.1] implies $\operatorname{ct}\left(M\left(\mathbb{Z}_{k}, 1\right)\right) \leq i+3 k$.

By combining Theorem 4.1, and Propositions 4.4 and 4.6 we obtain an upper bound for the covering type of Moore spaces with finitely generated homology. In the next 
result we will assume that there is at least some torsion in homology, since the torsionfree case is settled by Theorem 4.1 .

Corollary 4.7 Assume $n \geq 1$. Then

$$
\operatorname{ct}\left(M\left(\mathbb{Z}^{r} \oplus \mathbb{Z}_{k_{1}} \oplus \ldots \oplus \mathbb{Z}_{k_{n}}, i\right)\right) \leq i+k_{0}+3\left(k_{1}+\cdots+k_{n}\right)-2(n-1),
$$

where $k_{0}=\min \left\{k \geq 0 \mid\left(\begin{array}{c}i+k \\ i+1\end{array}\right) \geq r\right\}$.

Proof We are going to estimate the covering type of

$$
M\left(\mathbb{Z}^{r}, i\right) \vee M\left(\mathbb{Z}_{k_{1}}, i\right) \vee \cdots \vee M\left(\mathbb{Z}_{k_{n}}, i\right),
$$

which can be taken as a model for $M\left(\mathbb{Z}^{r} \oplus \mathbb{Z}_{k_{1}} \oplus \cdots \oplus \mathbb{Z}_{k_{n}}, i\right)$. By Theorem 4.1 we have $\operatorname{ct}\left(M\left(\mathbb{Z}^{r}, i\right)\right)=i+k_{0}+1$. Since $M\left(\mathbb{Z}^{r}, i\right)$ is $i$-dimensional and $M\left(\mathbb{Z}_{k_{1}}, i\right)$ is $(i+1)$-dimensional, Propositions 4.4 and 4.6 yield

$$
\operatorname{ct}\left(M\left(\mathbb{Z}^{r}, i\right) \vee M\left(\mathbb{Z}_{k_{1}}, i\right)\right) \leq\left(i+k_{0}+1\right)+\left(i+3 k_{1}\right)-i-1=i+k_{0}+3 k_{1}
$$

If we add more finite cyclic summands both terms in the wedge are $(i+1)$-dimensional, and so the covering type increases at most by $3 k_{j}-2$ at each step. Observe that the formula is valid even if $r=0$, because in that case $k_{0}=0$.

Acknowledgements We are very grateful to the referee for careful reading of the paper and for many valuable suggestions and comments which helped us to correct our arguments and improve considerably the manuscript.

Open Access This article is distributed under the terms of the Creative Commons Attribution 4.0 International License (http://creativecommons.org/licenses/by/4.0/), which permits unrestricted use, distribution, and reproduction in any medium, provided you give appropriate credit to the original author(s) and the source, provide a link to the Creative Commons license, and indicate if changes were made.

\section{References}

1. Arnoux, P., Marin, A.: The Kühnel triangulation of the complex projective plane from the view-point of complex cristallography. II. Mem. Fac. Sci. Kyushu Univ. Ser. A 45(2), 167-244 (1991)

2. Bagchi, B., Datta, B.: Minimal triangulations of sphere bundles over the circle. J. Combin. Theory Ser. A 115(5), 737-752 (2008)

3. Borghini, E., Minian, E.G.: The covering type of closed surfaces and minimal triangulations. J. Combin. Theory Ser. A 166, 1-10 (2019)

4. Brehm, U., Kühnel, W.: Combinatorial manifolds with few vertices. Topology 26(4), 465-473 (1987)

5. Cornea, O., Lupton, G., Oprea, J., Tanré, D.: Lusternik-Schnirelmann Category. Mathematical Surveys and Monographs, vol. 103. American Mathematical Society, Providence (2003)

6. Datta, B.: Minimal triangulations of manifolds. J. Indian Inst. Sci. 87(4), 429-449 (2007)

7. Dranishnikov, A.N., Katz, M.G., Rudyak, Yu.B.: Small values of the Lusternik-Schnirelmann category for manifolds. Geom. Topol. 12(3), 1711-1727 (2008)

8. Hatcher, A.: Algebraic Topology. Cambridge University Press, Cambridge (2002)

9. Karoubi, M., Weibel, C.: On the covering type of a space. Enseign. Math. 62(3-4), 457-474 (2016)

10. Lutz, F.H.: Triangulated manifolds with few vertices: combinatorial manifolds (2005). arXiv:math/0506372 
11. Whitehead, G.W.: Elements of Homotopy Theory. Graduate Texts in Mathematics, vol. 61. Springer, Berlin (1978)

Publisher's Note Springer Nature remains neutral with regard to jurisdictional claims in published maps and institutional affiliations. 